\title{
Dipole Oscillations in Fermionic Mixtures
}

\author{
S. Chiacchiera \\ Centro de Física Computacional, Department of Physics, \\ University of Coimbra, P-3004-516, Coimbra, Portugal \\ T. Macrì and A. Trombettoni \\ SISSA and INFN, Sezione di Trieste, via Beirut 2/4, I-34151, Trieste, Italy
}

\begin{abstract}
We study dipole oscillations in a general fermionic mixture: starting from the Boltzmann equation, we classify the different solutions in the parameter space through the number of real eigenvalues of the small oscillations matrix. We discuss how this number can be computed using the Sturm algorithm and its relation with the properties of the Laplace transform of the experimental quantities. After considering two components in harmonic potentials having different trapping frequencies, we study dipole oscillations in three-component mixtures. Explicit computations are done for realistic experimental setups using the classical Boltzmann equation without intra-species interactions. A brief discussion of the application of this classification to general collective oscillations is also presented.

PACS numbers: 67.85.Lm, 51.10.+y
\end{abstract}

\section{INTRODUCTION}

The study of collective modes is a major tool to characterize and unravel the effects of interparticle interactions in a broad range of physical systems and in particular in experiments of ultracold atoms, where the scattering length can be tuned through Feshbach resonances [1, 2]. For ultracold bosons the collective oscillation frequencies in single-component gases depend on the interaction energy and in multi-component gases they depend on both intra- and inter-species interactions; for ultracold fermions the $s$-wave intra-species scattering length vanishes due to Pauli principle and the collective modes depend on the interactions between the different components, so that their study is crucial in multi-component fermionic mixtures.

In the last decade, the impressive experimental progresses in trapping and controlling Fermi gases [3, 4] allowed their study in highly controllable setups: it became possible to lower the temperature to reveal degeneracy effects [5], tune the interactions to explore the BEC-BCS crossover [6 -12], superimpose optical lattices 13-16] and polarize two-component Fermi mixtures [17, 18]. Collective modes in two-component mixtures have also been experimentally studied 1924$]$.

Among these progresses, it recently became experimentally manageable to study three-component fermionic mixtures of ${ }^{6} \mathrm{Li}$ [25] and of ${ }^{6} \mathrm{Li}-{ }^{40} \mathrm{~K}$ [26, 27]. In 25] a degenerate Fermi gas consisting of three different hyperfine states of ${ }^{6} \mathrm{Li}$ was created: the three scattering lengths $a_{12}, a_{13}$ and $a_{23}$ are different and tunable; the collisional stability of the gas has been studied, showing rather long lifetimes when one scattering length is close to the unitary limit and the other two are small [25]. In [26] the interspecies Feshbach resonances of a mixture of ${ }^{6} \mathrm{Li}$ and ${ }^{40} \mathrm{~K}$ were studied: the collisional stability has been recently determined [27], showing that a small sample of ${ }^{40} \mathrm{~K}$ immersed in a two-component ${ }^{6} \mathrm{Li}$ mixture is stable with very low loss rates for large negative scattering lengths between the two ${ }^{6} \mathrm{Li}$.

These systems are particularly promising for several reasons: they offer a way to investigate new strongly interacting Fermi systems; thanks to their enlarged parameter space, they could present novel phases related to the formation of unconventional pairing and stability of trions [28 32]; moreover, the possibility of having many components with tunable interactions can be used to study mechanisms of color superconductivity [33] and, in perspective, to simulate parts of the QCD phase diagram 34.

A natural way to characterize multi-component fermionic mixtures is provided by the study of their modes. For two-component Fermi mixtures, collective oscillations have been investigated in several situations of experimental relevance [23, 35 42] (see more references in the reviews [3, 4]) and their behaviour studied when different parameters are varied: temperature, scattering length, polarization (i.e., the relative number of atoms of the components) and mass ratio. The variety of possible dynamical regimes grows when the number of components increases: quantized vortices of three-component Fermi mixtures with attractive interactions have been recently studied [4].

The goal of the present paper is to discuss a simple way to characterize the properties of the collective oscillations of a general multi-component mixture. Restricting to temperatures larger than the superfluid critical temperature (see discussion in Section II), we will describe the Fermi gas mixture using the Boltzmann equation, with no intra-species interaction. Introducing a Gaussian ansatz for the distribution function, the equations for the small oscillations are determined: a convenient way to classify the different regimes in the parameter space is based on the study of the eigenvalues of the small oscillations matrix. Once that the eigenvalues are found, the experimental quantities (like the center of mass positions for 
dipole oscillations) can be determined. The real part of the eigenvalues determines the damping of the quantities, while the imaginary part is related to the oscillations: we show that the number of peaks of the analytical continuation of the Laplace transform of the physical quantities gives the number of real solutions (at variance, the peaks of the Fourier transform do not give the number of complex solutions). One can then classify the solutions of the linearized Boltzmann equation through the number of real eigenvalues of the small oscillations matrix. A real root corresponds to an overdamped eigenmode, whereas complex ones correspond to oscillatory modes and purely imaginary to undamped oscillatory modes. The small oscillation matrix is real, therefore complex eigenvalues appear in pairs of complex conjugates and the number of oscillation frequencies is simply the number of pairs of complex roots. Counting the overdamped eigenmodes (i.e. the real roots), one has a measure of the degree of collectivity of the system: the larger this number, the stronger the effect of interactions and trap confinement in reducing the degrees of freedom of the mixture. We will show that this approach is complementary to the study of the oscillatory frequencies, and convenient to classify the properties of the small oscillations solutions. We will focus on the case of dipole oscillations (i.e, onedimensional out-of-phase motion of the centers of mass of the various components), but the proposed classification works for general collective modes of multi-component fermionic mixtures.

The plan of the paper is the following: In the next Section we review the formalism of the Boltzmann equation and the method of averages, applying it to a multicomponent fermionic mixture and deriving the equations of motion for the centers of mass of the components. In Section III we study the dipole oscillations of a twocomponent mixture in harmonic potentials having different trapping frequencies: indeed, when the trapping frequencies are equal, two of the eigenvalues of the $4 \times 4$ small oscillation matrix are purely imaginary due to the Kohn theorem [1, 2, 44], while for different trapping frequencies one can have either 0 or 2 or 4 real solutions, similarly to what happens for a three-component mixture with equal frequencies. In Section III we also recall that a convenient way to count the number of real eigenvalues is based on the Sturm theorem and we discuss how to characterize the different solutions starting from the experimental quantities. In Section IV we study dipole oscillations in three-component mixtures, presenting results for a mixture of three hyperfine levels of ${ }^{6} \mathrm{Li}$ and for a mixture of two hyperfine levels of ${ }^{6} \mathrm{Li}$ and one of ${ }^{40} \mathrm{~K}$, like in the recently reported experimental results [25 27]. In Sec. V we draw our conclusions and discuss possible further developments, whereas in the Appendices additional material is presented.

\section{BOLTZMANN EQUATION FOR A MULTI-COMPONENT MIXTURE}

In this Section we discuss the main properties of the Boltzmann equation for a trapped multi-component fermionic mixture. Consider a trapped balanced twocomponent Fermi gas; when the temperature $T$ is significantly larger than the Fermi temperature $T_{F}$, the gas behaves classically and is in the collisionless regime. Decreasing $T$, the frequency of collisions increases leading to a hydrodynamical behaviour. Further decreasing the temperature $T$ (but with $T$ larger than the superfluid critical temperature), degeneracy effects become strong and one would expect the mixture to be collisionless again. For weak (inter-species) interactions, this sequence collisionless-hydrodynamical-collisionless actually occurs as $T / T_{F}$ decreases from values larger than one towards smaller ones. However, if the interactions are strong (i.e., close to the unitary limit) the sequence found in the experiments [22, 23] is collisionlesshydrodynamical as it would be in a classical gas (i.e, without degeneracy effects): the gas remains hydrodynamical. This finding can be explained by taking into account in-medium effects: the in-medium enhancement of the cross-section compensates Pauli blocking at low temperature leading to a hydrodynamical behaviour [23, 42]. In other words, classical statistics seems to work well when interactions are strong because Pauli blocking and in-medium effects almost cancel each other. In summary, depending on the interaction strength, either degeneracy dominates or is compensated by medium effects and the low temperature behaviour is different in the two cases; however, whatever the interaction strength is, for temperatures above $\sim 0.5 T_{F}$ the Boltzmann equation framework with no intra-species interactions and classical collision term with no medium-effects for the inter-species interactions is found to work quite well, not only qualitatively, in reproducing the collective modes [23, 42].

We will take advantage of this finding, and describe the Fermi nature of the particles simply by forbidding intra-species interactions. In multi-component imbalanced mixtures each component has its own Fermi temperature $T_{F, \alpha}$, therefore we expect our scheme to work well above $\sim 0.5 \max \left\{T_{F, \alpha}\right\}$.

If we restrict ourselves to the normal phase (that is, for temperatures above the critical temperature for superfluidity), each component of the mixture can be described by a semiclassical distribution function $f_{\alpha}=f_{\alpha}(\mathbf{r}, \mathbf{p}, t)$ obeying the Boltzmann equation [45]. Here and in the following we denote the $N$ components of the multicomponent mixture by $\alpha=1, \cdots, N$, the $\alpha$-th component having $N_{\alpha}$ atoms of mass $m_{\alpha}$. The function $f_{\alpha}(\mathbf{r}, \mathbf{p}, t)$ gives the probability of finding a particle of species $\alpha$ at time $t$ in the $d^{3} r d^{3} p$ volume centered in $(\mathbf{r}, \mathbf{p})$ in phase space. The normalization condition of $f_{\alpha}$ is

$$
\int d \Gamma f_{\alpha}(\mathbf{r}, \mathbf{p}, t)=N_{\alpha}, \quad \text { with } \quad d \Gamma \equiv d^{3} r d^{3} p,
$$


and the density is

$$
\rho_{\alpha}(\mathbf{r}, t)=\int d^{3} p f_{\alpha}(\mathbf{r}, \mathbf{p}, t) .
$$

The Boltzmann equation for our multi-component mixture reads

$$
\frac{\partial f_{\alpha}}{\partial t}+\dot{\mathbf{r}}_{\alpha} \cdot \frac{\partial f_{\alpha}}{\partial \mathbf{r}}+\dot{\mathbf{p}}_{\alpha} \cdot \frac{\partial f_{\alpha}}{\partial \mathbf{p}}=-\sum_{\gamma=1}^{N} I_{\alpha \gamma}
$$

where

$$
\dot{\mathbf{p}}_{\alpha}=-\frac{\partial \mathcal{V}_{\alpha}}{\partial \mathbf{r}}, \quad \dot{\mathbf{r}}_{\alpha}=\frac{\mathbf{p}}{m_{\alpha}} .
$$

The right-hand side of Boltzmann equation (3) is a sum of collision integrals and the ' on the sum means that we sum on all $\gamma \neq \alpha . \mathcal{V}_{\alpha}$ is the single particle potential felt by the $\alpha$ species. In general, different components feel different anisotropic trapping potentials:

$$
\mathcal{V}_{\alpha}(\mathbf{r}) \equiv \frac{m_{\alpha}}{2}\left(\omega_{\alpha x}^{2} x^{2}+\omega_{\alpha y}^{2} y^{2}+\omega_{\alpha z}^{2} z^{2}\right)
$$

(we will not consider in the following the case in which the harmonic potentials $\mathcal{V}_{\alpha}$ have different centers [46]).

The collision integrals for classical statistics read [45]

$$
I_{\alpha \gamma} \equiv \int d^{3} p_{1} d \Omega \frac{d \sigma_{\alpha \gamma}}{d \Omega}\left|\frac{\mathbf{p}}{m_{\alpha}}-\frac{\mathbf{p}_{1}}{m_{\gamma}}\right|\left(f_{\alpha} f_{\gamma 1}-f_{\alpha}^{\prime} f_{\gamma 1}^{\prime}\right)
$$

and represent the variation of $f_{\alpha}$ due to collisions with particles of type $\gamma$. In the latter $\frac{d \sigma_{\alpha \gamma}}{d \Omega}$ is the differential cross section of an $\alpha$ atom and a $\gamma$ atom in the center of mass of the collision and $\Omega$ is the angle between the relative outgoing and relative ingoing momenta of the two colliding particles (in our model there is no intraspecies interaction: $\left.d \sigma_{\alpha \alpha}=0\right)$. The atoms $\alpha$ and $\gamma$ have respectively momenta $\mathbf{p}$ and $\mathbf{p}_{1}$ before the scattering and $\mathbf{p}^{\prime}$ and $\mathbf{p}_{1}^{\prime}$ after: momentum and kinetic energy are conserved in the collision $\left[\mathbf{p}+\mathbf{p}_{\mathbf{1}}=\mathbf{p}^{\prime}+\mathbf{p}_{1}^{\prime}\right.$ and $\left.p^{2} / 2 m_{\alpha}+p_{1}^{2} / 2 m_{\gamma}=p^{2} / 2 m_{\alpha}+p_{1}^{\prime 2} / 2 m_{\gamma}\right]$ and all the $f$ are evaluated at the same point and time, but different momenta: $f_{\alpha}=f_{\alpha}(\mathbf{r}, \mathbf{p}, t), f_{\gamma 1}=f_{\gamma}\left(\mathbf{r}, \mathbf{p}_{1}, t\right)$, $f_{\alpha}^{\prime}=f_{\alpha}\left(\mathbf{r}, \mathbf{p}^{\prime}, t\right)$ and $f_{\gamma 1}^{\prime}=f_{\gamma}\left(\mathbf{r}, \mathbf{p}_{1}^{\prime}, t\right)$.

With the classical collision term given in Eq. (6) the equilibrium distribution function is the MaxwellBoltzmann distribution

$$
\bar{f}_{\alpha}(\mathbf{r}, \mathbf{p})=\frac{\exp \left\{-\beta\left[p^{2} /\left(2 m_{\alpha}\right)+\mathcal{V}_{\alpha}(\mathbf{r})-\mu_{\alpha}\right]\right\}}{(2 \pi \hbar)^{3}},
$$

where $\beta \equiv 1 / k_{B} T$ and the chemical potential is fixed by the normalization condition Eq. (11). For the harmonic trapping potential of Eq. (5) it is

$$
\mu_{\alpha}=\frac{1}{\beta} \ln \left[\left(\beta \bar{\omega}_{\alpha}\right)^{3} N_{\alpha}\right],
$$

where

$$
\bar{\omega}_{\alpha} \equiv\left(\omega_{\alpha x} \omega_{\alpha y} \omega_{\alpha z}\right)^{1 / 3}
$$

is the trap average frequency.

The effect of Fermi or Bose statistics (blocking or antiblocking of the final state of a collision) can be incorporated in the collision integral by adding appropriate factors: then, consistently, the equilibrium distribution function to which the system is driven by collisions is a Fermi or Bose one [45].

To study collective oscillations without directly solving the Boltzmann equation, it is useful to derive equations for integrated average quantities: defining the average of a generic quantity $\chi=\chi(\mathbf{r}, \mathbf{p})$ in the component $\alpha$ as

$$
\langle\chi\rangle_{\alpha} \equiv \frac{1}{N_{\alpha}} \int d \Gamma f_{\alpha}(\mathbf{r}, \mathbf{p}, t) \chi(\mathbf{r}, \mathbf{p}),
$$

from the Boltzmann equation (3) one finds

$$
\frac{d\langle\chi\rangle_{\alpha}}{d t}-\left\langle\frac{\mathbf{p}}{m_{\alpha}} \cdot \frac{\partial \chi}{\partial \mathbf{r}}\right\rangle_{\alpha}+\left\langle\frac{\partial \mathcal{V}_{\alpha}}{\partial \mathbf{r}} \cdot \frac{\partial \chi}{\partial \mathbf{p}}\right\rangle_{\alpha}=-\sum_{\gamma=1}^{N}\left\langle I_{\alpha \gamma} \chi\right\rangle_{\alpha},
$$

where we have defined the collisional average

$$
\left\langle I_{\alpha \gamma} \chi\right\rangle_{\alpha} \equiv \frac{1}{N_{\alpha}} \int d \Gamma I_{\alpha \gamma} \chi(\mathbf{r}, \mathbf{p}) .
$$

The collisional averages of a quantity $\chi$ satisfy the following constraint in each pair of components

$$
\begin{aligned}
& N_{\alpha}\left\langle I_{\alpha \gamma} \chi\right\rangle_{\alpha}+N_{\gamma}\left\langle I_{\gamma \alpha} \chi\right\rangle_{\gamma}=\frac{1}{2} \int d \Gamma d^{3} p_{1} d \Omega \frac{d \sigma_{\alpha \gamma}}{d \Omega} \\
& \times\left|\frac{\mathbf{p}}{m_{\alpha}}-\frac{\mathbf{p}_{1}}{m_{\gamma}}\right|\left(f_{\alpha} f_{\gamma 1}-f_{\alpha}^{\prime} f_{\gamma 1}^{\prime}\right) \Delta \chi,
\end{aligned}
$$

where $\Delta \chi \equiv \chi_{\alpha}+\chi_{\gamma 1}-\chi_{\alpha}^{\prime}-\chi_{\gamma 1}^{\prime}$ : summing the latter over $\alpha, \gamma$ one finds the following global constraint

$$
\begin{aligned}
& \sum_{\alpha, \gamma}^{\prime} N_{\alpha}\left\langle I_{\alpha \gamma} \chi\right\rangle_{\alpha}=\frac{1}{4} \sum_{\alpha, \gamma}^{\prime} \int d \Gamma d^{3} p_{1} d \Omega \frac{d \sigma_{\alpha \gamma}}{d \Omega} \\
& \times\left|\frac{\mathbf{p}}{m_{\alpha}}-\frac{\mathbf{p}_{1}}{m_{\gamma}}\right|\left(f_{\alpha} f_{\gamma 1}-f_{\alpha}^{\prime} f_{\gamma 1}^{\prime}\right) \Delta \chi .
\end{aligned}
$$

If $\chi$ is a collisional invariant, i.e., if $\Delta \chi=0$ [45], then

$$
\sum_{\alpha, \gamma}{ }^{\prime} N_{\alpha}\left\langle I_{\alpha \gamma} \chi\right\rangle_{\alpha}=0
$$

Eq. (11) gives rise to a set of equations involving different observables: the goal is to have a closed set of equations to solve. This approach has been used to determine in a classical gas the shift of the collective frequencies due to the interparticle collisions in a Bose-Einstein condensate above the condensation temperature [47 50] and it has been applied to the study of the scissor mode in twocomponent Fermi mixtures [39] as well as to Fermi-Bose mixtures [51, 52]. A convenient way to evaluate the collisional averages to close the set of equations and study the small oscillations for the linearized Boltzmann equation is to make a Gaussian ansatz for the time-dependent 
distribution function $f$ : in [47] the frequency and the damping of quadrupole oscillation in a classical gas obtained using the Gaussian ansatz was compared with the numerical solution of the Boltzmann equation showing a good agreement.

For dipole oscillations along the direction $x$, choosing $\chi=x, p_{x}$ from Eq. (11) one gets the following $2 N$ equations

$$
\left\{\begin{array}{l}
\frac{d\langle x\rangle_{\alpha}}{d t}-\frac{\left\langle p_{x}\right\rangle_{\alpha}}{m_{\alpha}}=-\sum_{\gamma=1}^{N}{ }^{\prime}\left\langle I_{\alpha \gamma} x\right\rangle_{\alpha} \\
\frac{d\left\langle p_{x}\right\rangle_{\alpha}}{d t}+m_{\alpha} \omega_{\alpha x}^{2}\langle x\rangle_{\alpha}=-\sum_{\gamma=1}^{N}{ }^{\prime}\left\langle I_{\alpha \gamma} p_{x}\right\rangle_{\alpha},
\end{array}\right.
$$

with $\alpha=1, \cdots, N$. To close the set of Eqs. (15), adapting the method of [47] we make the following Gaussian ansatz for the distribution function:

$$
f_{\alpha}(\mathbf{r}, \mathbf{p}, t)=\frac{\exp \left\{-\beta\left[\frac{\left(\mathbf{p}-\mathbf{p}_{\alpha}\right)^{2}}{2 m_{\alpha}}+\mathcal{V}_{\alpha}\left(\mathbf{r}-\mathbf{r}_{\alpha}\right)-\mu_{\alpha}\right]\right\}}{(2 \pi \hbar)^{3}},
$$

where $\mathbf{p}_{\alpha}=\mathbf{p}_{\alpha}(t)$ and $\mathbf{r}_{\alpha}=\mathbf{r}_{\alpha}(t)$ depend on time. Eq. (16) is a local equilibrium ansatz: it generalizes (7) by giving it a time-dependent average coordinate $\left[\langle\mathbf{r}\rangle_{\alpha}=\mathbf{r}_{\alpha}(t)\right]$ and average momentum $\left[\langle\mathbf{p}\rangle_{\alpha}=\right.$ $\left.\mathbf{p}_{\alpha}(t)\right]$; moreover, it preserves the normalization condition $\left(\int d \Gamma f_{\alpha}(\mathbf{r}, \mathbf{p}, t)=N_{\alpha}\right)$.

Using the Gaussian ansatz (16) and linearizing the Eqs. (15) (i.e., retaining only terms that are at most linear in $\langle x\rangle_{\alpha}$ and $\left.\left\langle p_{x}\right\rangle_{\alpha}\right)$, one finds a closed set of equations. Indeed the collisional averages in the right-hand side of Eqs. (15) can be evaluated giving

$$
\begin{aligned}
& \left\langle I_{\alpha \gamma} x\right\rangle_{\alpha}=0 \\
& \left\langle I_{\alpha \gamma} p_{x}\right\rangle_{\alpha}=\frac{\mu_{\alpha \gamma}}{\tau_{\alpha \gamma}} N_{\gamma}\left(\frac{\left\langle p_{x}\right\rangle_{\alpha}}{m_{\alpha}}-\frac{\left\langle p_{x}\right\rangle_{\gamma}}{m_{\gamma}}\right)
\end{aligned}
$$

where $\mu_{\alpha \gamma}$ is the two body reduced mass $\left(\mu_{\alpha \gamma} \equiv\right.$ $m_{\alpha} m_{\gamma} / m_{\alpha \gamma}$, with $\left.m_{\alpha \gamma} \equiv m_{\alpha}+m_{\gamma}\right)$ : the explicit computation is reviewed in Appendix A. In Eq. (17b $\tau_{\alpha \gamma}$ is a parameter related to the collisions between atoms $\alpha$ and $\gamma$ and will turn out to be proportional to the relaxation time of the dipole mode: $\tau_{\alpha \gamma}$ is defined as

$$
\frac{1}{\tau_{\alpha \gamma}} \equiv \frac{4 \beta^{2} \hbar^{2}}{3 \pi}\left(\frac{\bar{\omega}_{\alpha} \bar{\omega}_{\gamma}}{\bar{\omega}_{\alpha \gamma}}\right)^{3} f\left(y_{\alpha \gamma}\right)
$$

where $y_{\alpha \gamma} \equiv \frac{\hbar^{2} \beta}{2 \mu_{\alpha \gamma} a_{\alpha \gamma}^{2}}$, with $a_{\alpha \gamma}$ the scattering length between the species $\alpha$ and $\gamma$. The function $f$ is defined in Eq. A4 and at the unitary limit $f=1 ; \bar{\omega}_{\alpha}$ is defined in Eq. (9) and we also used the notation

$$
\bar{\omega}_{\alpha \gamma} \equiv \prod_{i=x, y, z}\left(\frac{m_{\alpha} \omega_{\alpha i}^{2}+m_{\gamma} \omega_{\gamma i}^{2}}{m_{\alpha \gamma}}\right)^{1 / 6}
$$

Notice that repeating the derivation including Fermi statistics one would still get the equations Eqs. (17), the only modification being in the temperature dependence of the parameter $\tau_{\alpha \gamma}$.

Equations 15, together with Eqs. (17), form a closed set of differential equations describing the small oscillations of the centers of mass of a multi-component fermionic mixture at $T \gtrsim 0.5 \max \left\{T_{F, \alpha}\right\}$.

\section{A. Two components with equal trapping frequencies}

As a simple application of Eqs. (15) we retrieve in this Section the well-known case of a two-component mixture having equal trapping frequencies along the dipole motion direction: $\omega_{1 x}=\omega_{2 x} \equiv \omega_{x}$. One easily checks that the coordinate and velocity of the center of mass of the mixture decouple from the relative position and velocity and oscillate with frequency $\omega_{x}$, according to Kohn theorem. By defining the (average) relative position and velocity respectively as $x_{\text {rel }} \equiv\langle x\rangle_{1}-\langle x\rangle_{2}$ and $v_{\text {rel }} \equiv\left\langle p_{x}\right\rangle_{1} / m_{1}-\left\langle p_{x}\right\rangle_{2} / m_{2}$ one gets

$$
\left\{\begin{array}{l}
\frac{d x_{\mathrm{rel}}}{d t}-v_{\mathrm{rel}}=0 \\
\frac{d v_{\mathrm{rel}}}{d t}+\omega_{x}^{2} x_{\mathrm{rel}}=-\frac{1}{\tau^{\prime}} v_{\mathrm{rel}}
\end{array}\right.
$$

where the inverse relaxation time is $\frac{1}{\tau^{\prime}}=\frac{M_{1}+M_{2}}{m_{12}} \frac{1}{\tau_{12}}$ and $M_{\alpha} \equiv N_{\alpha} m_{\alpha}$ is the total mass of the component $\alpha$. It follows that if

$$
\frac{1}{\tau^{\prime}}>2 \omega_{x}
$$

then the relative motion is overdamped: as long as the interactions are larger than a critical value the transport of momentum is so effective that the two components cannot move independently.

\section{TWO COMPONENTS WITH DIFFERENT TRAPPING FREQUENCIES}

In this Section we consider a mixture of two components in potentials having different trapping frequencies $\left(\omega_{1 x}, \omega_{1 y}, \omega_{1 z}\right)$ and $\left(\omega_{2 x}, \omega_{2 y}, \omega_{2 z}\right)$. This case deserves a separate discussion since the properties of the solutions of the small oscillation equations are similar to those of a three-component mixture with equal trapping frequencies: when the trapping frequencies are equal along the direction of the dipole motion $\left(\omega_{1 x}=\omega_{2 x}\right)$, two of the eigenvalues of the $4 \times 4$ small oscillation matrix are purely imaginary due to the Kohn theorem [1, 2, 44], while for different trapping frequencies $\left(\omega_{1 x} \neq \omega_{2 x}\right)$ one can have either 0 or 2 or 4 real solutions, as it happens for a threecomponent mixture. In this Section we also apply the Sturm theorem to count the number of real eigenvalues of the small oscillation matrix and we discuss how to characterize the solutions according to this number starting from the experimental quantities. We finally discuss the 


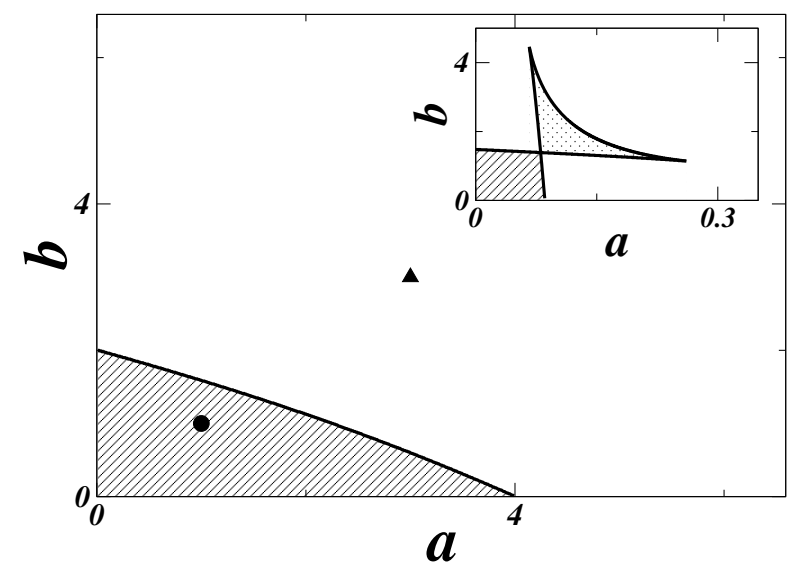

FIG. 1: Plot of the regions having 0 (dashed) and 2 (blank) real roots in the plane $a, b\left(a=\frac{M_{1}}{m_{12}} \frac{1}{\tau \omega_{1 x}}, b=\frac{M_{2}}{m_{12}} \frac{1}{\tau \omega_{1 x}}\right)$ for the case $c=\frac{\omega_{2 x}^{2}}{\omega_{1 x}^{2}}=4$. Inset: plot of regions having 0 (dashed), 2 (blank) and 4 (dotted) real roots for the case $c=0.001$.

properties of the Fourier and Laplace transforms of the center of mass positions giving informations respectively on the imaginary and real part of the eigenvalues.

We define the dimensionless quantities $X_{\alpha}(t) \equiv$ $\langle x\rangle_{\alpha}(t) / L$ and $V_{\alpha}(t) \equiv\left\langle p_{x}\right\rangle_{\alpha}(t) /\left(m_{\alpha} \omega_{1 x} L\right)(\alpha=1,2$ and $L$ is a length scale of the system which can be set equal to an harmonic oscillator length); time will be scaled in units of $1 / \omega_{1 x}$. The equations of motion for the dimensionless centers of mass positions $X_{\alpha}$ and velocities $V_{\alpha}$ are then

$$
\left\{\begin{array}{l}
\dot{X}_{1}=V_{1} \\
\dot{X}_{2}=V_{2} \\
\dot{V}_{1}=-X_{1}-b\left(V_{1}-V_{2}\right) \\
\dot{V}_{2}=-c X_{2}+a\left(V_{1}-V_{2}\right),
\end{array}\right.
$$

where $a \equiv \frac{M_{1}}{m_{12}} \frac{1}{\tau \omega_{1 x}}, b \equiv \frac{M_{2}}{m_{12}} \frac{1}{\tau \omega_{1 x}}, c \equiv \frac{\omega_{2 x}^{2}}{\omega_{1 x}^{2}}$ : the parameter $\tau$ is defined in Eq. (18) and $M_{\alpha} \equiv N_{\alpha} m_{\alpha}$ is the total mass of the component $\alpha$.

It is convenient to introduce a matrix notation for the equations of motion: defining the vector $\mathbf{Y} \equiv$ $\left(X_{1}, X_{2}, V_{1}, V_{2}\right)^{T}$ we can write Eqs. (21) as

$$
\dot{\mathbf{Y}}=G_{2} \mathbf{Y},
$$

where the $4 \times 4$ matrix $G_{2}$ is given by

$$
G_{2}=\left(\begin{array}{cccc}
0 & 0 & 1 & 0 \\
0 & 0 & 0 & 1 \\
-1 & 0 & -b & b \\
0 & -c & a & -a
\end{array}\right)
$$

Of course, once that the eigenvalues $\lambda_{1}, \cdots, \lambda_{4}$ of the matrix (23) have been determined, it is possible to ob-
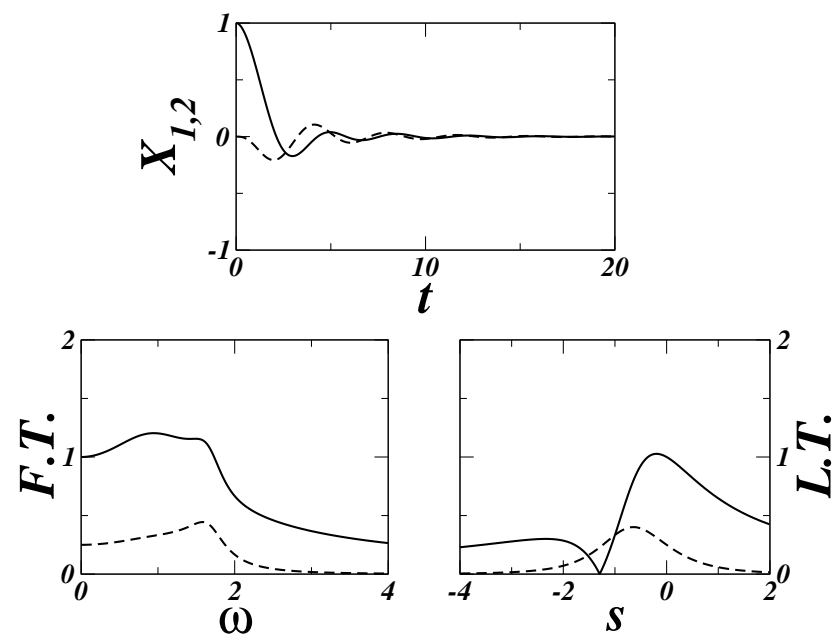

FIG. 2: Top: Plot of the center of mass $X_{1}$ (solid line) and $X_{2}$ (dashed line) as a function of time for $a=1, b=1$ (circle in Fig 1). Bottom left: modulus of the Fourier transform of $X_{1,2}$, given by (27). Bottom right: modulus of the Laplace transform of $X_{1,2}$ defined in (29). Initial conditions: $X_{1}(0)=$ $1, X_{2}(0)=0, V_{1}(0)=V_{2}(0)=0 ; c=4$ as in Fig 1

tain the time evolution of the centers of mass. If $\mathcal{B}$ is the matrix diagonalizing $G_{2}$ such that $\mathcal{B}^{-1} G_{2} \mathcal{B}=$ $\operatorname{diag}\left(\lambda_{1}, \cdots, \lambda_{4}\right)$, one has

$$
\mathbf{Y}_{j}(t)=\sum_{k=1}^{4} c_{j k} e^{\lambda_{k} t}
$$

where the coefficients $c_{j k}$ depend upon the initial conditions through the relation $c_{j k}=\mathcal{B}_{j k} \sum_{\ell=1}^{4} \mathcal{B}_{k \ell}^{-1} \mathbf{Y}_{\ell}(0)$.

The properties of the solutions of Eqs. (22) are completely determined by the properties of the eigenvalues $\lambda_{k}$ of the matrix (23), which are given by the roots of the equation

$$
P(\lambda)=\lambda^{4}+(a+b) \lambda^{3}+(1+c) \lambda^{2}+(a+b c) \lambda+c=0,
$$

where $P(\lambda)$ is the characteristic polynomial of the matrix $G_{2}$. Since $P(\lambda)$ is a polynomial with real coefficients, if it has a complex root it has also as a root its complex conjugate: this means that Eq. (25) has either 0 or 2 or 4 real solutions. It is easy to verify that the solutions of the equation $P(\lambda)=0$ satisfy the property

$$
\lambda_{k}^{(R)} \leq 0,
$$

which just states the stability of solutions (see Appendix B): the real parts of the eigenvalues $\lambda_{k}$ correspond to the damping of the normal modes and the imaginary parts to their frequencies. In Eq. (26), as well as in the following, we denote the real part and the imaginary part of $\lambda_{k}$ respectively by $\lambda_{k}^{(R)}$ and $\lambda_{k}^{(I)}$. 
One has that $\lambda_{k}^{(R)}=0$ if and only if $c=1$ (i.e., $\omega_{1 x}=$ $\left.\omega_{2 x}\right)$ : indeed if $\left.c=1, P(\lambda)=\left(\lambda^{2}+1\right)\left[\lambda^{2}+(a+b) \lambda+1\right)\right]$ and two solutions are just $\pm i$. These two roots correspond to the oscillations of the center of mass of the mixture with frequency equal to the trap one and without damping, as discussed in Section II A. Then for equal trapping frequencies Eq. (25) can have only 0 or 2 real solutions, and the critical value for passing from one region to the other is just given by Eq. (201).

To compute the number of real solutions we could look at the general solution of the fourth degree equation (25); however, in order to generalize this approach to three (or more) components it is more convenient and straightforward to use the Sturm algorithm [53], which we briefly recall in Appendix B. In Fig 1 we plot the regions with 0, 2 and 4 real roots in the plane $a, b$ for two values of $c$; the inset shows that in some narrow region of the parameter space ( $c=0.001$ in the inset) regions with 4 real roots may occur.

If we adopt the complementary point of view of counting the number of independent oscillations (the number of pairs of complex solutions) we see that: only for equal trapping frequencies $(c=1)$ we have an undamped oscillatory mode (Kohn mode); on the contrary, for unequal frequencies $(c \neq 1)$, all the modes are either damped or overdamped. The number of the damped oscillatory modes is 2,1 or 0 , corresponding then to 0,2 or even 4 real roots of Eq. (25).

Fourier and Laplace transforms. We now discuss how to determine the regions with a different number of real roots from experimentally measured quantities. When the centers of mass of the two components have been determined or measured, informations on the real and imaginary parts of the eigenvalues $\lambda_{k}$ can be obtained respectively from their Fourier and Laplace transforms:

$$
\begin{gathered}
F_{\alpha}(\omega) \equiv \int_{0}^{\infty} e^{-i \omega t} X_{\alpha}(t) d t \\
L_{\alpha}(s) \equiv \int_{0}^{\infty} e^{-s t} X_{\alpha}(t) d t .
\end{gathered}
$$

Each pair of complex roots, say $\lambda_{1}=\lambda_{1}^{(R)}+i \lambda_{1}^{(I)}$ and $\lambda_{2}=\lambda_{1}^{(R)}-i \lambda_{1}^{(I)}$, having an imaginary part $\pm i \lambda_{1}^{(I)}$, should correspond to a maximum of the modulus of the Fourier transform at $\omega=\lambda_{k}^{(I)}$ : then, one could expect that if the Fourier transform has (for positive values of $\omega)$ two distinct peaks there should be 4 complex eigenvalues (i.e., 0 real solutions). However, Fourier transforms of the centers of mass can be analytically computed for arbitrary initial conditions, showing that two peaks (at two positive values of $\omega$ ) do not always occur when Eq. (25) has 4 complex roots: e.g., if the positions at which the two peaks should be are too close, the peaks merge into one. Therefore one cannot easily infer the number of complex solutions from the peaks of the Fourier transforms.
On the other side, to each real solution corresponds a pole in the Laplace transform when $s$ is extended to negative values: indeed, setting $X_{\alpha}(t) \equiv \sum_{k=1}^{4} C_{k} e^{\lambda_{k} t}$ with the coefficients $C_{k}$ depending upon the initial conditions, one has for $s>0$ the analytical expression

$$
L_{\alpha}^{(a n .)}(s)=\sum_{k=1}^{4} \frac{C_{k}}{s-\lambda_{k}} .
$$

Eq. (29) can be defined also for negative values $s$ and it has a pole when $s$ is equal to a real (and negative) eigenvalue.

One can experimentally measure $X_{\alpha}(t)$ : of course, to determine the eigenvalues $\lambda_{k}$ one can directly fit the data with the expression $X_{\alpha}(t)=\sum_{k=1}^{4} C_{k} e^{\lambda_{k} t}$. In particular, since the number of real eigenvalues is 0,2 or 4 , one can use as fitting functions the sum of 4 exponentials (corresponding to 4 real eigenvalues), or the sum of 2 exponentials and an exponential modulated by a sinusoidal (corresponding to 2 real and 2 complex conjugate eigenvalues), or the sum of 2 exponentials modulated by sinusoidals (corresponding to 4 complex eigenvalues, i.e. 2 pairs of complex conjugate eigenvalues): the best fit among them would determine the number of real eigenvalues. Another (possibly complementary) method to determine the eigenvalues $\lambda_{k}$ is to extend the definition (28) for $L_{\alpha}(s)$ to negative values of $s$. One has that the integral in (28) is defined for $s>s_{\min }=-\min \left\{\left|\lambda_{k}^{(R)}\right|\right\}$. Therefore the Laplace transform of the center of mass position has a divergence at $s=s_{\text {min }}$ : if $\lim _{s \rightarrow s_{\text {min }}}\left(s-s_{\text {min }}\right) L_{\alpha}(s)=0$, then $s_{\min }$ is the real part of a pair of complex conjugate roots (say, $\lambda_{1}$ and $\lambda_{2}$ ) and one has to fit from data the corresponding imaginary part $\lambda_{1}^{(I)}$ (with $\lambda_{2}^{(I)}=-\lambda_{1}^{(I)}$ ) and coefficients $C_{1,2}$; if not, then $s_{\text {min }}$ is a real root (say, $\left.\lambda_{1}\right)$ and $\lim _{s \rightarrow s_{\text {min }}}\left(s-s_{\text {min }}\right) L_{\alpha}(s)=C_{1}$. In the latter case, one can define a quantity $\tilde{X}_{\alpha}(t) \equiv X_{\alpha}(t)-C_{1} e^{\lambda_{1} t}$ : again, from the divergence of the Laplace transform of $\tilde{X}_{\alpha}(t)$ for negative values of $s$ one can determine the next eigenvalue [similarly, for a complex root one has to define $\left.\tilde{X}_{\alpha}(t) \equiv X_{\alpha}(t)-C_{1} e^{\lambda_{1} t}-C_{2} e^{\lambda_{2} t}\right]$.

The resulting behaviour of the centers of mass positions and of the modulus of the Fourier transform (27) and the Laplace transform (29) is plotted in Fig2 and Fig 3 for two sets of parameters, one belonging to the region of zero and one in the region of two real eigenvalues: the Laplace transforms show respectively zero and two sharp peaks.

\section{THREE COMPONENTS}

In this Section we discuss the properties of the modes of three-component mixtures: we consider three-component fermionic mixtures of ${ }^{6} \mathrm{Li}$, as in the setup reported in [25], and mixtures with two ${ }^{6} \mathrm{Li}$ and one ${ }^{40} \mathrm{~K}$ species [26, 27]. We focus for sake of simplicity on isotropic potentials 
$\mathcal{V}_{\alpha}$ with the same trapping frequency $\omega$. Rescaling the variables as in Section III, measuring time in units of $1 / \omega$ and defining the vector $\mathbf{Y}=\left(X_{1}, X_{2}, X_{3}, V_{1}, V_{2}, V_{3}\right)^{T}$ we have $\dot{\mathbf{Y}}=G_{3} \mathbf{Y}$, where the $6 \times 6$ matrix $G_{3}$ is:

$G_{3}=\left(\begin{array}{cccc}\mathbf{0} & \multicolumn{4}{c}{} \\ & -\Gamma_{12}-\Gamma_{13} & \Gamma_{12} & \Gamma_{13} \\ -\mathbb{1} & \Gamma_{21} & -\Gamma_{21}-\Gamma_{23} & \Gamma_{23} \\ & \Gamma_{31} & \Gamma_{32} & -\Gamma_{31}-\Gamma_{32}\end{array}\right)$ where $\mathbf{0}$ and $\mathbb{1}$ are the $3 \times 3$ zero and identity matrices and $\Gamma_{\alpha \gamma} \equiv \frac{M_{\gamma}}{m_{\alpha \gamma}} \frac{1}{\omega \tau_{\alpha \gamma}}\left(1-\delta_{\alpha \gamma}\right)(\alpha, \gamma=1,2,3)$ with $\delta_{\alpha \gamma}$ the Kronecker delta. The six non-zero parameters $\Gamma_{\alpha \gamma}$ are not independent: they satisfy the relation $\Gamma_{12} \Gamma_{23} \Gamma_{31}=$ $\Gamma_{13} \Gamma_{32} \Gamma_{21}$. The characteristic polynomial is:

$$
P(\lambda)=\left(\lambda^{2}+1\right)\left[\lambda^{4}+\lambda^{3} \sum_{\alpha, \gamma} \Gamma_{\alpha \gamma}+\lambda^{2}\left(2+\sum_{\alpha, \gamma, \epsilon \neq \alpha} \Gamma_{\alpha \gamma} \Gamma_{\gamma \epsilon}+\frac{1}{2} \sum_{\alpha, \gamma, \epsilon \neq \alpha} \Gamma_{\alpha \gamma} \Gamma_{\epsilon \gamma}\right)+\lambda \sum_{\alpha, \gamma} \Gamma_{\alpha \gamma}+1\right]
$$

The discussion proceeds now as in Section III: one has

- $\lambda_{k}^{(R)} \leq 0$ (Stability of solutions)

- $\lambda_{k}^{(R)}=0 \Leftrightarrow \lambda_{k}^{(I)}= \pm 1$,

the number of real solutions can be either 0,2 or 4 and the number of real roots can be inferred from the Laplace transforms of experimental measured quantities. At the transition line between one region with 0 or 2 real eigenvalues and a region having more real roots, $\delta$ functions appear and the corresponding strengths of these $\delta$ functions become non-vanishing. The eigenvalues $\lambda= \pm i$ correspond to the center of mass oscillation with the frequency of the trap (Kohn theorem). Again, with different trapping frequencies, regions with 6 real eigenvalues (that is, with no oscillatory mode at all) may occur.

\section{A. Three-component mixture of the same species}

We discuss in this Section the behaviour of the eigenvalues of a mixture in which the components belong to the same species, as in the three-component ${ }^{6} \mathrm{Li}$ mixture described in [25].

For an unpolarized mixture in which each species contains an equal number of particles $N_{L i}\left(3 N_{L i}\right.$ is the total number of particles in the mixture), there are three independent parameters $\Gamma_{\alpha \gamma}\left(\right.$ since $\left.\Gamma_{\alpha \gamma}=\Gamma_{\gamma \alpha}\right)$ and the matrix $G_{3}$ reads

$$
G_{3}=\left(\begin{array}{cccc}
\mathbf{0} & \multicolumn{4}{c}{} \\
& -\left(\xi_{1}+\xi_{2}\right) & \xi_{1} & \xi_{2} \\
-\mathbb{1} & \xi_{1} & -\left(\xi_{1}+\xi_{3}\right) & \xi_{3} \\
& \xi_{2} & \xi_{3} & -\left(\xi_{2}+\xi_{3}\right)
\end{array}\right)
$$
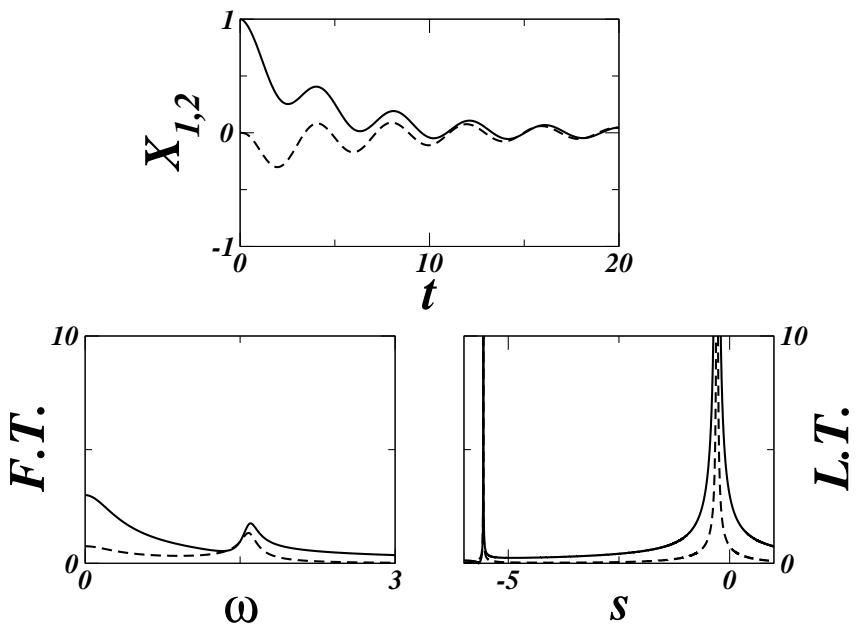

FIG. 3: Top: Plot of the center of mass $X_{1}$ (solid line) and $X_{2}$ (dashed line) as a function of time for $a=3, b=3$ (triangle in Fig(1). Bottom left: modulus of the Fourier transform of $X_{1,2}$. Bottom right: modulus of the Laplace transform of $X_{1,2}$ defined in (29). Initial conditions: $X_{1}(0)=1, X_{2}(0)=0$, $V_{1}(0)=V_{2}(0)=0 ; c=4$ as in Fig 1

where $\xi_{1} \equiv \Gamma_{12}, \xi_{2} \equiv \Gamma_{13}$ and $\xi_{3} \equiv \Gamma_{23}$. The characteristic equation and its solutions are studied in Appendix B.

In Fig 4 we plot the results of our analysis with the Sturm theorem in the plane $\xi_{1}-\xi_{2}$ for two values of $\xi_{3}$. If $\xi_{3}<1$, then one has three different regions corresponding to 0,2 and 4 real solutions. When $\xi_{3}>1$ the region with 0 real solutions disappears and the motion is ruled either by one or two oscillating frequencies, but not three. 

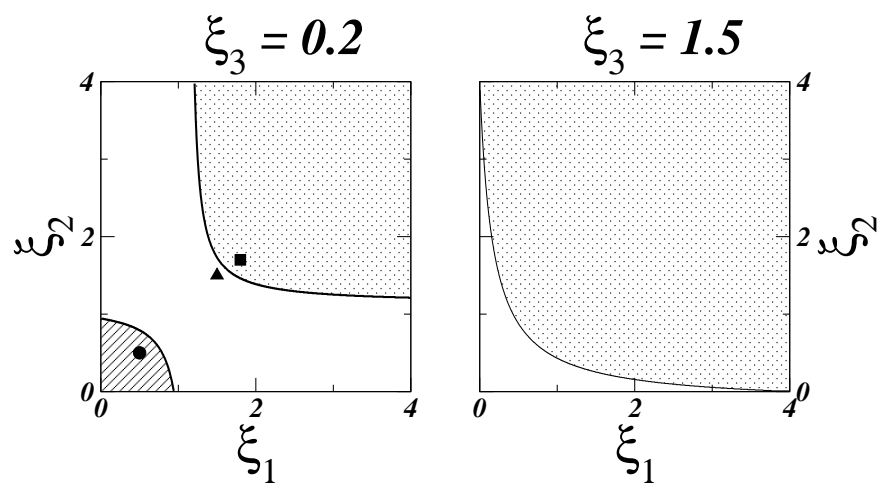

FIG. 4: Plot in the $\xi_{1}-\xi_{2}$ plane of regions having 0 (dashed), 2 (blank) and 4 (dotted) real roots for a three-component mixture having $\xi_{3}=0.2$ (left) and $\xi_{3}=1.5$ (right) - the value of $\xi_{3}$ at which the region with 0 real roots disappears is $\xi_{3}=1$. The points denoted in the left plot are at $\xi_{1}=0.5, \xi_{2}=0.5$ (circle), $\xi_{1}=1.5, \xi_{2}=1.5$ (triangle), $\xi_{1}=1.8, \xi_{2}=1.7$ (square).

In Fig 5 the center of mass positions for a simple initial condition and the modulus of their Fourier and Laplace (29) transforms are plotted for three sets of parameters, belonging to regions with respectively 0,2 and 4 real eigenvalues showing that the Laplace transforms display respectively zero, two and four sharp peaks.

When the mixture is polarized, one has to use Eq. (30): varying the number of the atoms in the components, it is possible to explore the different regions through the polarization. An example is shown in Fig 6 , where the number of atoms of the component 3 is varied and the components 1 and 2 are at the unitary limit. As discussed in [25], the mixture is collisionally stable for small values of the other scattering lengths. Fig 6 shows that by varying the trapping frequency one can explore the three regions with 0,2 and 4 real eigenvalues. Notice that the stronger the confinement due to the trap, the fewer independent oscillatory modes are possible.

\section{B. Different species}

In this Section we present the results for a threecomponent mixture of two species of ${ }^{6} \mathrm{Li}$ (denoted by $L i_{1}$ and $L i_{2}$ ) and one species of ${ }^{40} K$ recently realized experimentally: a small sample of ${ }^{40} K$ is immersed in a two-component ${ }^{6} \mathrm{Li}$ mixture and found to be collisionally stable with very low loss rates for large negative scattering lengths between the two ${ }^{6} L i$ (for $\left|a_{L i-L i}\right| \gtrsim 1400 a_{0}$, where $a_{0}$ is the Bohr radius) [27]. The matrix $G_{3}$ of Eq.
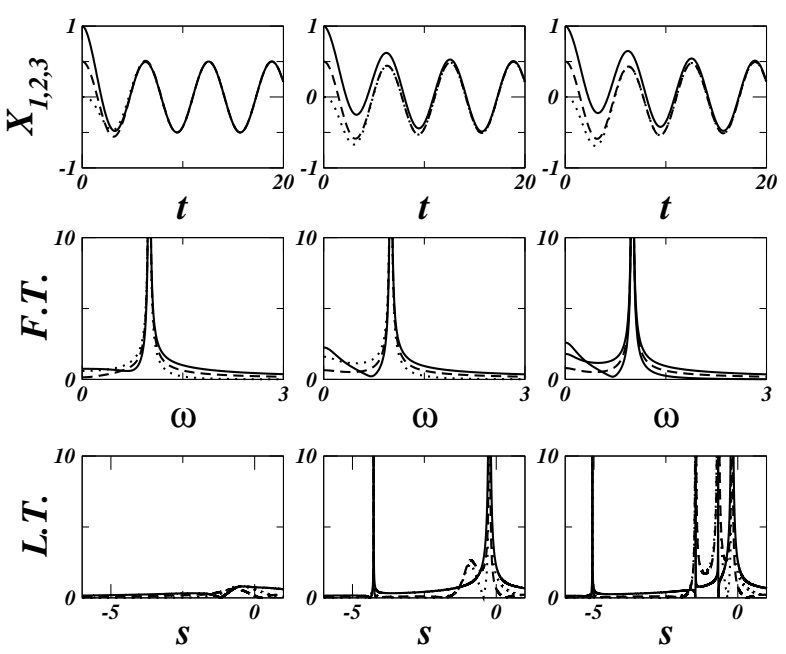

FIG. 5: Plot of the center of mass $X_{1,2,3}(t)$ (top row), modulus of the Fourier transform $F_{1,2,3}(\omega)$ (central row) and modulus of the Laplace transform $L_{1,2,3}(s)$ defined in (29) (bottom row). The left, central and right columns correspond respectively to the point $\xi_{1}=0.5, \xi_{2}=0.5$ (circle in Fig 4) - 0 real roots), $\xi_{1}=1.5, \xi_{2}=1.5$ (triangle -2 real roots), $\xi_{1}=1.8$, $\xi_{2}=1.7$ (square -4 real roots). Solid, dashed and dotted lines are for the corresponding quantities of the species 1,2 and 3. Initial conditions: $X_{1}(0)=1, X_{2}(0)=0.5, X_{3}(0)=0$, $V_{1}(0)=V_{2}(0)=V_{3}(0)=0$.

(30) simplifies to

$$
G_{3}=\left(\begin{array}{cccc}
\mathbf{0} & & \mathbb{1} & \\
& & & \\
& -\left(\xi_{1}+\xi_{2} r \alpha\right) & \xi_{1} & \xi_{2} r \alpha \\
-\mathbb{1} & \xi_{1} & -\left(\xi_{1}+\xi_{2} r \alpha\right) & \xi_{2} r \alpha \\
& \xi_{2} & \xi_{2} & -2 \xi_{2}
\end{array}\right)
$$

where we defined $\xi_{1} \equiv \Gamma_{12}, \xi_{2} \equiv \Gamma_{31}, r \equiv m_{K} / m_{L i}$ and $\alpha \equiv N_{K} / N_{L i}$. To simplify formulas, we assumed the same number of atoms in the two $\mathrm{Li}$ species and equal isotropic trapping frequencies, but similar (even if more involved) expressions can be found for the realistic case of anisotropic cigar-shaped potentials and with the trapping frequencies of $L i_{1}-L i_{2}$ different from those of the $K$ component: they will be reported elsewhere.

In Fig 7 we plot the number of real solutions taking a mixture with the same number of particles for the two Lithium components and a smaller number of particles of the $K$ component. We vary the scattering length between $L i_{1}$ and $L i_{2}$ and the trapping frequency $\omega$.

The number of oscillatory modes is reduced, again, by increasing the interaction strength or making the trap more confining. 


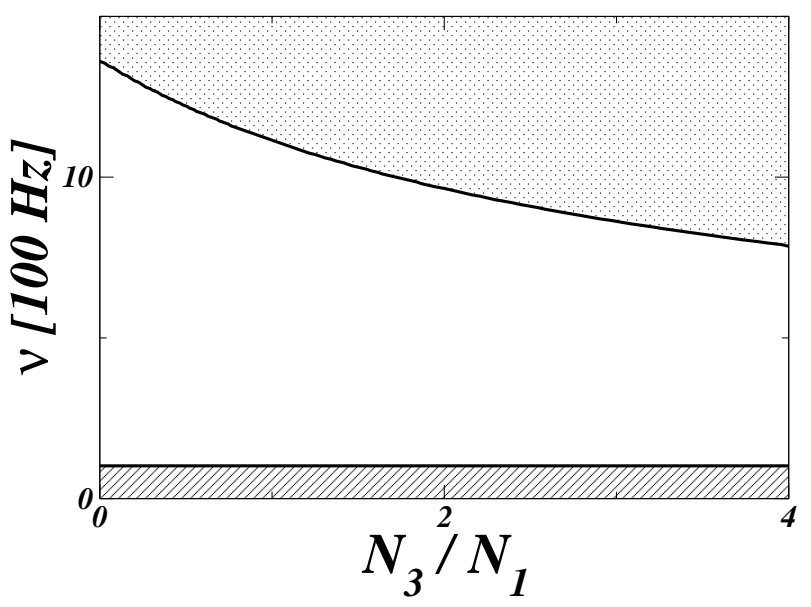

FIG. 6: Plot of regions having 0 (dashed), 2 (blank) and 4 (dotted) real roots for a mixture of three $L i$ species when the number $N_{3}$ and the trapping frequency $\omega=2 \pi \nu$ are varied. The components 1 and 2 are taken at unitary limit with a fixed number of atoms $N_{1}=N_{2}=5 \cdot 10^{4}$. Temperature is fixed $(T=500 n K)$ and the two remaining scattering lengths are chosen to be $a_{13}=a_{23}=400 a_{0}$ (similar plots are obtained when $a_{13}$ and $a_{23}$ are different, but small).

\section{CONCLUSIONS}

In this paper we used the Boltzmann equation to study dipole oscillations in a general fermionic mixture: to discuss and classify the different solutions in the parameter space through it, we used the number of real eigenvalues of the small oscillations matrix, discussing how this number is related to the Laplace transform of the experimental quantities. The small oscillation matrix for a $\mathrm{N}$ component mixture has $2 N$ eigenvalues and the complex ones appear in pairs of complex conjugates: the number of independent collective mode frequencies is therefore given by $(2 N-$ number of real roots $) / 2$. Counting the real roots (overdamped modes) and the complex ones (oscillatory ones) are complementary descriptions. The reason why we chose the first one is to take advantage of Sturm algorithm and Laplace transform properties.

After deriving the equations of motion for the centers of mass of the components and discussing as a simple example the case of two components in harmonic potentials having different trapping frequencies, we studied dipole oscillations in three-component mixtures.

A $N$-component mixture where each component experiences a different trapping has a maximum of $N$ independent oscillation frequencies. We found, as expected, that when interactions are favoured (by increasing the strength of interactions between components, or shrinking the traps) the components tend to move together and the number of independent frequencies reduces. If all

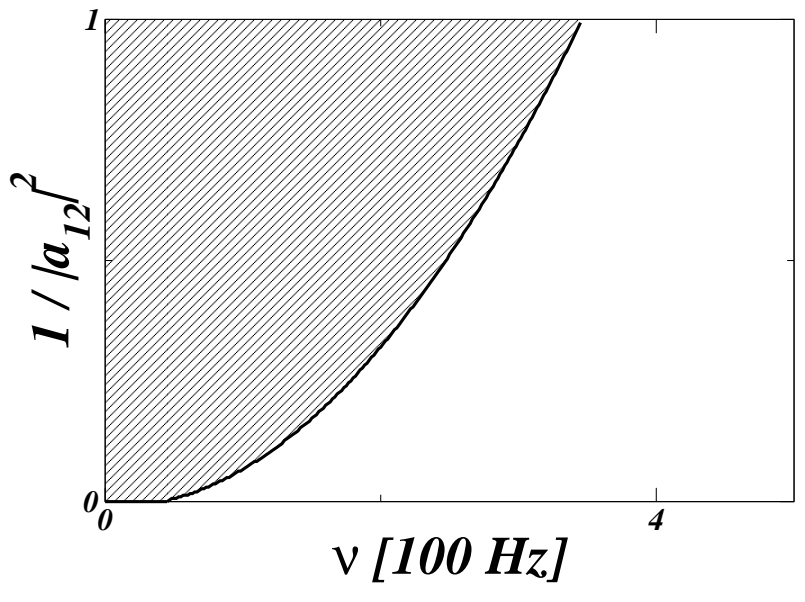

FIG. 7: Plot of regions having 0 (dashed) and 2 (blank) real roots for a mixture of $L i_{1}-L i_{2}-K$ with equal trapping frequencies $\omega=2 \pi \nu$ and equal number of $L i$ atoms $N_{L i_{1}}=$ $N_{L i_{2}} \equiv N_{L i}=5 \cdot 10^{4}$. The number of $K$ atoms has been chosen $N_{K}=0.25 N_{L i}$. Parameters: $T=200 \mathrm{nK}$, $a_{L i_{1}-K}=a_{L i_{2}-K}=63 a_{0}$; the $L i_{1}-L i_{2}$ scattering length $a_{12}$ is measured in units of $100 a_{0}$. For $\nu<55 \mathrm{~Hz}$ there are 0 real roots whatever $a_{12}$ is.

the trap frequencies are equal along one direction there is one undamped oscillation with frequency equal to the trap one (Kohn theorem). Beside that, there can be up to $N-1$ damped oscillatory modes. On the contrary, if the trap frequencies are different, there is no purely oscillatory mode. The number of damped oscillatory modes can be at most $N$ and can be reduced down to 0 : it is possible that there is no collective dipole oscillatory mode at all in the mixture.

In explicit computations we used the Boltzmann equation with classical collision terms and without intraspecies interactions: this gives, for strong inter-species interactions, results very close to the findings obtained including Fermi statistics and in-medium effects thanks to a compensation of effects 23, 42]. Using the classical Boltzmann equation without intra-species interactions is expected to be a good approximation (also far from the unitary limit) at temperatures above $\sim 0.5 \max \left\{T_{F, \alpha}\right\}$, where $T_{F, \alpha}$ is the Fermi temperature of the component $\alpha$ : for two components, e.g., it well reproduces the experimental results for the scissor mode for these temperatures 23,42$]$. We also observe that the explicit inclusion of the Fermi statistics modifies the dependence of the relaxation times upon the system parameters, but not the dynamical equations for the centers of mass and their momenta and then the subsequent analysis based on the Sturm theorem would proceed in the same way.

The focus of this paper has been on the study of dipole oscillations, however we point out that a similar study 
can be done for different (and more complicated) collective modes: one has to write the small oscillations equations, and determine the number of real roots of the small oscillations matrix.

To conclude, we observe that multi-component mixtures are promising to study mechanisms of color superconductivity [33] due to the possibility of having many components with tunable interactions: from this point of view, to study the collective oscillations (and follow the modifications and the fate of the regions in the parameter space discussed in this paper) when the temperature is lowered until the superfluid critical temperature can provide useful informations on possible intermediate, new strongly correlated phases.

\section{ACKNOWLEDGEMENTS}

We thank for fruitful discussions M. Urban, S. Jochim, F. Schreck and S. Moroni. This work is supported by the grants INSTANS (from ESF) and 2007JHLPEZ (from MIUR).

\section{Appendix A: Computation of $\tau$}

In this appendix we give some details on the computation of the parameter $\tau$ related to the collisions between atoms of species 1 and atoms of species 2. As usual, given two particles with masses $m_{1}, m_{2}$, positions $\mathbf{r}_{1}, \mathbf{r}_{2}$ and momenta $\mathbf{p}_{1}, \mathbf{p}_{2}$ the global and relative coordinate and momenta are defined as: $\mathbf{P} \equiv \mathbf{p}_{1}+\mathbf{p}_{2}, \mathbf{R} \equiv\left(m_{1} \mathbf{r}_{1}+\right.$ $\left.m_{2} \mathbf{r}_{2}\right) / m_{12}, \mathbf{r}_{\text {rel }} \equiv \mathbf{r}_{1}-\mathbf{r}_{2}, \mathbf{p}_{\text {rel }} \equiv\left(m_{2} \mathbf{p}_{1}-m_{1} \mathbf{p}_{2}\right) / m_{12}$. Then $p_{1}^{2} / 2 m_{1}+p_{2}^{2} / 2 m_{2}=P^{2} / 2 m_{12}+p_{\text {rel }}^{2} / 2 \mu$ and $\mathbf{p}_{\text {rel }}=$ $\mu \dot{\mathbf{r}}_{\text {rel }}$, where $\mu \equiv \frac{m_{1} m_{2}}{m_{1}+m_{2}}$ is the reduced mass and $m_{12} \equiv$ $m_{1}+m_{2}$. For simplicity we consider equal isotropic trapping frequencies: $\omega_{1, i}=\omega_{2, i} \equiv \omega(i=x, y, z)$; $a_{12}$ is the scattering length and $\frac{d \sigma}{d \Omega}=\frac{1}{p_{\text {rel }}^{2} / \hbar^{2}+1 / a_{12}^{2}}$ the differential cross section. The collisional average $\left\langle I_{12} p_{x}\right\rangle_{1}$ is defined as

$$
\left\langle I_{12} p_{x}\right\rangle_{1}=\frac{1}{N_{1}} \int d \Gamma I_{12} p_{x}
$$

Plugging the Gaussian ansatz for the distribution function (16) in Eq. (A1) and moving to the center of mass and relative coordinates we obtain

$$
\left\langle I_{12} p_{x}\right\rangle_{1}=\frac{2 \beta^{4} \omega^{3}}{3 \pi} \frac{N_{2} v_{\mathrm{rel}}}{\mu} \int_{0}^{\infty} d p_{\mathrm{rel}} \frac{p_{\mathrm{rel}}^{5}}{\frac{p_{\mathrm{rel}}^{2}}{\hbar^{2}}+\frac{1}{a_{12}^{2}}} e^{\frac{-\beta p_{\mathrm{rel}}^{2}}{2 \mu}}
$$

where $\mu$ is the reduced mass and $v_{\text {rel }}$ the relative velocity between the two components. Setting $\frac{\beta}{2 \mu} p_{\text {rel }}^{2}=t$ then we have:

$$
\left\langle I_{12} p_{x}\right\rangle_{1}=\frac{4 \hbar^{2} \beta^{2} \omega^{3}}{3 \pi} N_{2} v_{\mathrm{rel}} \mu \int_{0}^{\infty} d t \frac{t^{2}}{t+y} e^{-t}
$$

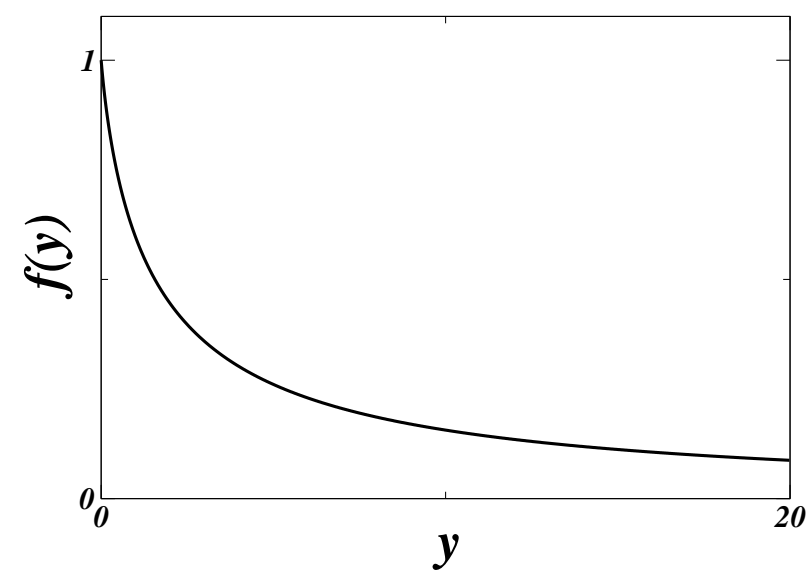

FIG. 8: Plot of the function $f(y)$ defined in Eq. A4 as a function of the dimensionless parameter $y=\hbar^{2} \beta / 2 \mu a_{12}^{2}$.

where $y=\frac{\hbar^{2} \beta}{2 \mu a_{12}^{2}}$. The last integral in the right-hand side of (A3) can be written in terms of the generalized $\Gamma$-function $\Gamma(0, x)=\int_{x}^{\infty} d t \frac{e^{-t}}{t}[\underline{54}]$ as

$$
f(y)=\int_{0}^{\infty} d t \frac{t^{2}}{t+y} e^{-t}=1-y+y^{2} e^{y} \Gamma(0, y) .
$$

The function $f(y)$ is plotted in Fig, 8 , it is $f(0)=1$ (at the unitary limit) and $f$ vanishing for large $y$. One then obtains

$$
\frac{1}{\tau}=\frac{4 \beta^{2} \hbar^{2} \omega^{3}}{3 \pi} f(y)
$$

The computation for general anisotropic and different trapping frequencies can be similarly done and gives the result (18).

\section{Appendix B: Sturm chains and Sturm theorem.}

Here we recall how to apply Sturm theorem to count the number of real roots of a polynomial equation $P(\lambda)=$ 0 [53]. First, one has to define the (finite) sequence of polynomials (Sturm chain) as follows:

$$
\begin{aligned}
P_{0}(\lambda) & \equiv P(\lambda) \\
P_{1}(\lambda) & \equiv P^{\prime}(\lambda) \\
P_{2}(\lambda) & \equiv-\operatorname{rem}\left(P_{0}(\lambda), P_{1}(\lambda)\right) \\
P_{3}(\lambda) & \equiv-\operatorname{rem}\left(P_{1}(\lambda), P_{2}(\lambda)\right) \\
\cdots & \equiv \cdots \\
0 & =-\operatorname{rem}\left(P_{m-1}(\lambda), P_{m}(\lambda)\right)
\end{aligned}
$$


where we denote with $\operatorname{rem}\left(P_{m-1}(\lambda), P_{m}(\lambda)\right)$ the rest of the polynomial division of the polynomials $P_{m-1}(\lambda)$ and $P_{m}(\lambda)$. Then one has to evaluate the sign of the polynomials $P_{i}(\lambda)$ at $+\infty$ and $-\infty$ and to compute the number of changes of sign (which we call $\Delta(+\infty)$ and $\Delta(-\infty)$ ) in the sequences $\left\{P_{0}(+\infty), P_{1}(+\infty), P_{2}(+\infty), \ldots\right\}$ and $\left\{P_{0}(-\infty), P_{1}(-\infty), P_{2}(-\infty), \ldots\right\}$. Sturm theorem states that the number of real solutions of the equation $P(x)=$ 0 is given by $|\Delta(+\infty)-\Delta(-\infty)|$. Notice that the Sturm theorem is not directly applicable to cases where there are degenerate solutions. In these cases, one has first to transform the equation into one that has only simple roots, but this can always be done.

We show how to apply Sturm theorem in the specific case of polynomial (25) of fourth degree

$$
P(\lambda)=\lambda^{4}+\lambda^{3}(a+b)+\lambda^{2}(1+c)+\lambda(a+b c)+c .
$$

In order to evaluate the sign of Sturm polynomials at $\lambda= \pm \infty$ we determine the sign of the coefficient of the highest power in the polynomial as follows:

\begin{tabular}{c|ccccc}
$\lambda$ & $P_{0}$ & $P_{1}$ & $P_{2}$ & $P_{3}$ & $P_{4}$ \\
\hline$+\infty$ & + & + & $(-1)^{\alpha}$ & $(-1)^{\beta}$ & $(-1)^{\gamma}$ \\
\hline$-\infty$ & + & - & $(-1)^{\alpha}$ & $-(-1)^{\beta}$ & $(-1)^{\gamma}$
\end{tabular}

where $\alpha, \beta, \gamma=\{0,1\}$. Then number of changes of signs in the two cases is:

$\Delta(+\infty)=\frac{3}{2}-\frac{1}{2}\left[(-1)^{\alpha}+(-1)^{\alpha}(-1)^{\beta}+(-1)^{\beta}(-1)^{\gamma}\right]$
$\Delta(-\infty)=\frac{5}{2}+\frac{1}{2}\left[(-1)^{\alpha}+(-1)^{\alpha}(-1)^{\beta}+(-1)^{\beta}(-1)^{\gamma}\right]$ and the function counting the number of real solutions is

$$
|\Delta(+\infty)-\Delta(-\infty)|=\left|1+(-1)^{\alpha}+(-1)^{\alpha}(-1)^{\beta}+(-1)^{\beta}(-1)^{\gamma}\right| .
$$

\begin{tabular}{|c|c|c|}
\hline & $\alpha=0$ & $\alpha=1$ \\
\hline & $\gamma=0 \quad \gamma=1$ & $\gamma=0 \quad \gamma=1$ \\
\hline$\beta=0$ & 4 & 0 \\
\hline$\beta=1$ & 0 & 0 \\
\hline
\end{tabular}

The counting function ( $\mathrm{B} 3 \mathrm{l})$ is then
When the real part of the root of Eq. (B2) is negative, then solutions of equations of motion (21) do not grow exponentially: writing $P(\lambda)=\lambda^{4}+A \lambda^{3}+B \lambda^{2}+C \lambda+D$ this happens if [55]

$$
\begin{aligned}
& A>0 \quad B>0 \quad C>0 \quad D>0 \\
& A B C>A^{2} D+C^{2} .
\end{aligned}
$$

It is easy to verify that these conditions are satisfied.

The characteristic polynomial of the matrix $G_{3}$ in Eq.(32) is:

$$
\begin{aligned}
P(\lambda)= & \left(\lambda^{2}+1\right)\left\{\lambda^{4}+2 \lambda^{3}\left(\xi_{1}+\xi_{2}+\xi_{3}\right)\right. \\
& +\lambda^{2}\left[2+3\left(\xi_{1} \xi_{2}+\xi_{2} \xi_{3}+\xi_{1} \xi_{3}\right)\right] \\
& \left.+2 \lambda\left(\xi_{1}+\xi_{2}+\xi_{3}\right)+1\right\} .
\end{aligned}
$$

For completeness we write here the explicit solutions of the quartic equation $P(\lambda) /\left(\lambda^{2}+1\right)=0$ :

$$
\begin{aligned}
\lambda_{I, I I} & =\frac{1}{2}\left(-S-\sqrt{Q_{1}} \pm \sqrt{-4+Q_{2}+2 S \sqrt{Q_{1}}}\right) \\
\lambda_{I I I, I V} & =\frac{1}{2}\left(-S-\sqrt{Q_{1}} \pm \sqrt{-4+Q_{2}-2 S \sqrt{Q_{1}}}\right)
\end{aligned}
$$

where

$$
\begin{aligned}
S\left(\xi_{1}, \xi_{2}, \xi_{3}\right) & \equiv \xi_{1}+\xi_{2}+\xi_{3} \\
Q_{1}\left(\xi_{1}, \xi_{2}, \xi_{3}\right) & \equiv \xi_{1}^{2}+\xi_{2}^{2}+\xi_{3}^{2}-\xi_{1} \xi_{2}-\xi_{2} \xi_{3}-\xi_{1} \xi_{3} \\
Q_{2}\left(\xi_{1}, \xi_{2}, \xi_{3}\right) & \equiv 2\left(\xi_{1}+\xi_{2}+\xi_{3}\right)^{2}-3\left(\xi_{1} \xi_{2}+\xi_{2} \xi_{3}+\xi_{1} \xi_{3}\right)
\end{aligned}
$$

[1] L. P. Pitaevskii and S. Stringari, Bose-Einstein condensation (Oxford, Oxford University Press, 2003).

[2] C. J. Pethick and H. Smith, Bose-Einstein condensation in dilute gases, 2nd ed. (Cambridge, Cambridge University Press, 2008).

[3] W. Ketterle and M. W. Zwierlein, in Ultracold Fermi Gases, Proceedings of the international school held at Villa Monastero, Varenna, 20-30 June, 2006 eds. M. Inguscio, W. Ketterle, and C. Salomon (Amsterdam, IOS Press, 2007).

[4] S. Giorgini, L. P. Pitaevskii, and S. Stringari, Rev. Mod. Phys. 80, 1215 (2008).

[5] B.DeMarco and D.S. Jin, Science 285, 1703 (1999).

[6] M. Greiner, C. A. Regal, and D. S. Jin, Nature 426, 537 (2003).

[7] S. Jochim, M. Bartenstein, A. Altmeyer, G. Hendl, C. Chin, J. H. Denschlag, and R. Grimm, Science 302, 2101
(2003).

[8] M. W. Zwierlein, C. A. Stan, C. H. Schunck, S. M. F. Raupach, S. Gupta, Z. Hadzibabic, and W. Ketterle, Phys. Rev. Lett. 91, 250401 (2003).

[9] T. Bourdel, L. Khaykovich, J. Cubizolles, J. Zhang, F. Chevy, M. Teichmann, L. Tarruell, S. J. J. M. F. Kokkelmans, and C. Salomon, Phys. Rev. Lett 93, 050401 (2004).

[10] G. B. Partridge, K. E. Strecker, R. I. Kamar, M. W. Jack, and R. G. Hulet, Phys. Rev. Lett 95, 020404 (2005).

[11] J. Kinast, A. Turlapov, J. E. Thomas, Q. Chen, J. Stajic, and K. Levin, Science 307, 1296 (2005).

[12] G. Veeravalli, E. Kuhnle, P. Dyke, and C. J. Vale, Phys. Rev. Lett. 101, 250403 (2008)

[13] G. Modugno, F. Ferlaino, R. Heidemann, G. Roati, and M. Inguscio, Phys. Rev. A 68, 011601(R) (2003).

[14] J. K. Chin, D. E. Miller, Y. Liu, C. Stan, W. Setiawan, C. 
Sanner, K. Xu, and W. Ketterle, Nature 443, 961 (2006).

[15] T. Stöferle, H. Moritz, K. Günter, M. Köhl, and T. Esslinger, Phys. Rev. Lett. 96, 030401 (2006).

[16] U. Schneider, L. Hackermuller, S. Will, Th. Best, I. Bloch, T. A. Costi, R. W. Helmes, D. Rasch, and A. Rosch, Science 322, 1520 (2008).

[17] M. W. Zwierlein, A. Schirotzek, C. H. Schunck and W. Ketterle, Science 311, 492 (2006)

[18] G. B. Partridge, W. Li, R. I. Kamar, Y. A. Liao, R. G. Hulet, Science 311, 503 (2006).

[19] S. D. Gensemer and D. S. Jin, Phys. Rev. Lett. 87, 173201 (2001).

[20] J. Kinast, S. L. Hemmer, M. E. Gehm, A. Turlapov, and J. E. Thomas, Phys. Rev. Lett. 92, 150402 (2004).

[21] M. Bartenstein, A. Altmeyer, S. Riedl, S. Jochim, C. Chin, J. H. Denschlag, and R. Grimm, Phys. Rev. Lett. 92, 203201 (2004).

[22] M. J. Wright, S. Riedl, A. Altmeyer, C. Kohstall, E. R. S. Guajardo, J. H. Denschlag, and R. Grimm, Phys. Rev. Lett. 99, 150403 (2007).

[23] S. Riedl, E. R. SánchezGuajardo, C. Kohstall, A. Altmeyer, M. J. Wright, J. H. Denschlag, R. Grimm, G. M. Bruun, and H. Smith, Phys. Rev A 78, 053609 (2008).

[24] S. Nascimbène, N. Navon, K. J. Jiang, L. Tarruell, M. Teichmann, J. McKeever, F. Chevy, and C. Salomon, Phys. Rev. Lett. 103, 170402 (2009).

[25] T. B. Ottenstein, T. Lompe, M. Kohnen, A. N. Wenz, and S. Jochim, Phys. Rev. Lett. 101, 203202 (2008).

[26] E. Wille et al., Phys. Rev. Lett. 100, 053201 (2008).

[27] F. M. Spiegelhalder, A. Trenkwalder, D. Naik, G. Hendl, F. Schreck, and R. Grimm, Phys. Rev. Lett. 103, 223203 (2009).

[28] Á. Rapp, G. Zaránd, C. Honerkamp, and W. Hofstetter, Phys. Rev. Lett. 98, 160405 (2007).

[29] S. Capponi, G. Roux, P. Lecheminant, P. Azaria, E. Boulat, and S. R. White, Phys. Rev. A 77, 013624 (2008).

[30] X. W. Guan, M. T. Batchelor, C. Lee, and H.-Q. Zhou, Phys. Rev. Lett. 100, 200401 (2008).

[31] T. N. De Silva, Phys. Rev. A 80, 013620 (2009).

[32] P. F. Bedaquea and J. P. D'Incao, Ann. Phys. 324, 1763 (2009).

[33] M. G. Alford, A. Schmitt, K. Rajagopal, and T. Schäfer, Rev. Mod. Phys. 80, 1455 (2008).

[34] K. Rajagopal and F. Wilczek, At the frontier of particle physics, World Scientific, Vol. 1-3, Pages: 2062-
2151,(2001); arXiv:hep-ph/0011333.

[35] L. Vichi and S. Stringari, Phys. Rev. A 60, 4734 (1999).

[36] L. Vichi, J. Low Temp. Phys. 121, 177 (2000).

[37] G. M. Bruun and B. R. Mottelson, Phys. Rev. Lett. 87, 270403 (2001).

[38] M. Tomoyuki and G. F. Bertsch, Phys. Rev. A 73, 013610 (2006).

[39] G. M. Bruun and H. Smith, Phys. Rev. A 76, 045602 (2007).

[40] A. Lazarides and B. Van Schaeybroeck, Phys. Rev. A 77, 041602(R) (2008).

[41] H. P. Dahal, S. Gaudio, J. D. Feldmann, and K. S. Bedell, Phys. Rev. A 78, 035601 (2008).

[42] S. Chiacchiera, T. Lepers, D. Davesne, and M. Urban, Phys. Rev. A 79, 033613 (2009).

[43] G. Catelani and E. A. Yuzbashyan, arXiv:0909.3851

[44] W. Kohn, Phys. Rev. 123, 1242 (1961).

[45] G. E. Uhlenbeck, G. E. Ford, and E. W. Montroll, Lectures in statistical mechanics (Providence, RI, American Mathematical Society, 1963).

[46] J. Williams, R. Walser, J. Cooper, E. A. Cornell, and M. Holland, Phys. Rev. A 61, 033612 (2000).

[47] D. Guéry-Odelin, F. Zambelli, J. Dalibard, and S. Stringari, Phys. Rev. A 60, 4851 (1999).

[48] U. Al Khawaja, C. J. Pethick, and H. Smith, J. Low Temp. Phys. 118, 127 (2000).

[49] P. Pedri, D. Guéry-Odelin, and S. Stringari, Phys. Rev. A 68, 043608 (2003).

[50] W. J. Mullin and R. J. Ragan, Phys. Rev. A 74, 043607 (2006).

[51] G. Ferrari, M. Inguscio, W. Jastrzebski, G. Modugno, G. Roati, and A. Simoni, Phys. Rev. Lett. 89, 053202 (2002).

[52] F. Ferlaino, R. J. Brecha, P. Hannaford, F. Riboli, G. Roati, G. Modugno, and M. Inguscio, J. Opt. B: Quantum Semiclassical Opt. 5, S3 (2003).

[53] S. Basu, R. Pollack, and M. Roy, Algorithms in real algebraic geometry (Berlin, Springer, 2003).

[54] M. Abramowitz, I. A. Stegun, Handbook of mathematical functions with formulas, graphs, and mathematical tables (Dover, 1972, repr. ed).

[55] G. Birkhoff and S. MacLane, A survey of modern algebra (Macmillan, New York, 1953). 